the compressed as well as deviated spinal cord noticed at the lesional portion.

Although this method of examination was accompanied by the transcient headache to some extent and limited to the lateral view and the oblique view, the contrast medium used was the harmless air which was absorbed completely. Repeating of examination is possible. We emphasize that, especially in the cervical region, the air filled picture of the subarachnoideal space showed clearly and concretely the relationship between the cervical spine and spinal cord both statically and dynamically.

\title{
92. Conduction in Traumatic Cervical Syndrome
}

\author{
Masuo Kurihara, Taisuke Hayashi and Toru Hamada \\ Ist Department of Surgery, Wakayama Medical College
}

The maximum conduction velocity of the ulnar nerve was measured in patients suffering from traumatic cervical syndrome.

The ulnar nerve was stimulated both at elbow and wrist, and potencials evoked from $\mathrm{m}$. abductor digiti quinti were recorded. The maximum conduction velocity was calculated from the difference between latencies on the elbow and the wrist stimulation.

In cases with hypesthesia or increased tendonreflex of the upper extremity, the maximum conduction velocity revealed some facilitation. On the other hand, in cases with hyperesthesia or decreased tendonreflex of the upper extremity, the maximum conduction velocity showed some inhibition.

Thus it was concluded that the maximum conduction velocity varied according to the clinical features in the traumatic cervical syndrome.

\section{Studies on the Tinels Sign in Peripheral Nerve Regeneration}

\author{
Paul Tutomu Kotani, Yasushi Toyoshima, Kaichiro Nambu, Hideo \\ Matsuda, Yoshiaki Ishizaki, Takashi Suzuki, Hiroshi IWAMI, \\ Yoshiki Yamano, Masao Sasada, Hiroo Inove, \\ Haruhiko Azuma, Masaaki Hatanaka and Tôru Kuchrra \\ Department of Orthopedic Surgery, Osaka City University Medical School
}

It was attempted, in order to study the factors effecting to Tinels sign and the mechanism of this sign, to examine the sign on 20 nerves in 16 cases as follows. 